Results The most common reason for referral into the service was support adjusting to IBD and its symptoms (e.g. pain, fatigue, incontinence, tolerating uncertainty) (55\%), followed by anxiety (30\%) and depression (10\%). $75 \%$ of patients seen were female. We demonstrated improvements across a balanced scorecard of four dimensions: clinical effectiveness, service utilisation, patient satisfaction and referrer satisfaction.

Clinical effectiveness was measured through the use of validated questionnaires.

Short IBD Questionnaire (SIBD) scores pre- and post-psychological therapy showed significant reductions in symptoms across all domains $(p=0.003)$ with notable improvement in systemic symptoms (fatigue and energy levels) and emotional and social functioning. Statistically significant improvements $(\mathrm{p}=0.003)$ were seen in Patient Health Questionnaire (PHQ-9) depression score, and there was a strong trend of improvements in anxiety scores, using Generalised Anxiety Disorder Assessment (GAD-7) and quality of life $(p=0.061$ using EuroQoL). Patient and referrer satisfaction with the PSSPIBD was very high: $90 \%$ of patients and all referrers completing the feedback rated the service as excellent. Initial data comparing service use in one year before and after engaging with PSSPIBD found a statistically significantly reduction in outpatient appointments by $62.5 \%(\mathrm{p}=0.008)$ and in CT and MRI scans by $76.2 \%(\mathrm{p}=0.05)$.

Conclusions The pilot PSSPIBD demonstrated significant improvements across all domains, notably symptomatology and service utilisation. There should be increased access to integrated psychological support services with further evidence gathered of success across all domains.

\section{OWE-033 INTEGRATING MAJOR HAEMORRHAGE POLICIES: ENDOSCOPY AND LABORATORY WORKING TOGETHER} ${ }^{1}$ Julie Dwyer*, ${ }^{1}$ Audrey Murray, ${ }^{1}$ Lisa Law, ${ }^{2}$ Lesley MacDonald, ${ }^{2}$ Eleanor Hazra,
${ }^{2}$ Suzanne Whyte, ${ }^{2}$ Katherine Hands, ${ }^{1}$ Morag Barron, ${ }^{1}$ Max Groome. ${ }^{1}$ NHS Tayside, Dundee,
UK; ${ }^{2}$ East of Scotland Blood Transfusion Service, Dundee, UK

\subsection{6/gutjnl-2018-BSGAbstracts.449}

Introduction Major haemorrhage is a common medical emergency in endoscopy rooms where on site critical care support is not always standard. Successful management includes resuscitation, appropriate and safe use of blood products alongside effective communication between clinical and laboratory teams.

NICE, the National Patient Safety Association and the Serious Hazards of Transfusion organisation all specifically refer to the need for major haemorrhage protocols.

NHS Tayside's Blood Transfusion Service launched an updated major haemorrhage policy (MHP) in May 2017. We developed a protocol that combines practical clinical response within both the endoscopy room and the laboratory.

We wish to describe the development and implementation of this policy.

Methods A time and space study was completed looking at staff roles within the endoscopy room, typically endoscopist, nurse (RN) and Health Care Assistant (HCA); and the unit as a whole during major GI bleeds. Barriers to effective resuscitation, timely provision of blood products, communication with laboratories/other clinical teams were identified.

Results We identified variation in the following key areas:

1. Staff roles and numbers in room
2. Communication between nursing, medical and laboratory teams

3. Need for a team leader to free up the endoscopist

The following protocol was developed.

Endoscopist Declares major haemorrhage.

Assesses haemostasis/patients global status, requests additional clinical support (eg anaesthetics).

Nursing Room RN activates in-room buzzer triggering protocol.

Three additional RNs and 1 HCA supplement the original room team.

RN A (Team Leader) Activates Laboratory MHP via '2222' call, coordinates the room, allocates staff roles, runs resuscitation, communicates with additional teams

RN B - assists with therapeutics

RN C - patient observations, fluid/medication delivery, scribes

HCA A - runner for equipment etc

Porter - available throughout

Laboratory '2222' call triggers the laboratory and blood bank to prepare and dispatch: 4 units packed red cells, 4 units fresh frozen plasma and 1 pool of platelets directly to the endoscopy unit by a dedicated porter. The lab remains on standby as required.

The policy's introduction was supplemented by training sessions including simulated scenarios to increase staff awareness, confidence and responsiveness to major haemorrhage.

Conclusions We believe this protocol is the first to give a practical description of a process dovetailing clinical and laboratory response to major haemorrhage. Having a clearly defined team leader and standardising individual staff roles allows streamlined communication with other clinical groups in a non critical care environment.

\section{ADTU-09 RECRUITMENT AND RETENTION OF BLOOD DONORS AMONG PATIENTS WITH HAEMOCHROMATOSIS: 5 YEARS OF EXPERIENCE}

${ }^{1}$ Jennifer Gallacher*, ${ }^{2}$ Jill Clarkson, ${ }^{1,3}$ Steven Masson. 'Liver Transplant Unit, Newcastle Upon Tyne Hospitals, Newcastle, UK; ${ }^{2}$ NHS Blood and Transplant, Newcastle, UK; ${ }^{3}$ Institute of Cellular Medicine, Newcastle University Medical School, Newcastle, UK

\subsection{6/gutjnl-2018-BSGAbstracts.450}

Introduction In patients with haemochromatosis, the current standard treatment is by removal of iron by regular venesection. EASL guidelines recommend that, in uncomplicated haemochromatosis patients, blood from therapeutic venesection should be made available to national blood transfusion services. A scheme to facilitate blood donation in these patients was trialled, and here we describe 5 years' experience of this pilot.

Methods A specialist haemochromatosis clinic was developed. In this clinic, an information leaflet and self-referral application to the blood services were provided to those patients interested in becoming blood donors. These applications were countersigned by the responsible physician and upon receipt of the form, the local blood service team contacted patients to determine their eligibility. Data on new referrals and resultant donations from the clinic were collected.

Results Following implementation of this Haemochromatosis Pathway, 169 patients have been seen in the dedicated clinic (109 male; median age 56). The majority (156) had uncomplicated haemochromatosis. From these, 66 were potentially 